\title{
Extractive fermentation of Monascus purpureus promotes the production of oxidized congeners of the pigment azaphilone
}

\author{
Yoon Ji Lim ${ }^{1} \cdot$ Doh Won Lee ${ }^{1} \cdot$ Si-Hyung Park ${ }^{2} \cdot$ Hyung-Jin Kwon ${ }^{1}$ \\ Received: 27 September 2018 / Accepted: 13 October 2018 / Published Online: 31 December 2018 \\ (C) The Korean Society for Applied Biological Chemistry 2018
}

\begin{abstract}
Monascus is a source of food colorant with high productivity of the pigment azaphilone. Monascus azaphilone (MAz) is biosynthesized through a single non-reducing polyketide pathway, the major components of which are ankaflavin (1), monascin (2), rubropunctatin (3) and monascorubrin (4); valuable biological activities have been reported for these compounds. Thus, various culture conditions were explored to reduce the cost of culture ingredients, enhance productivity and modulate compound composition. In the present study, we examined an extractive fermentation (EF) method with Diaion HP-20 resin (HP20) in direct comparison to a previously explored method involving Triton X-100 (TX100) to explore the modulated production of the major MAzs. We employed wild-type Monascus purpureus as well as two derivative recombinant strains ( $\triangle m p p G$ and $\triangle m p p E)$ that are known to have differential MAz profiles as that of the wild-type strain. The HP20 resin was capable of modulating the MAz profile in favor of orange MAzs 3 and $\mathbf{4}$, oxidized congeners in this class, as was TX100-a phenomenon not previously observed for TX100 EF with Monascus anka. These finding substantiate that HP20 can be employed for the selective production of oxidized MAz and for diversifying the culture conditions used for Az production.
\end{abstract}

Keywords Diaion HP-20 resin · Extractive fermentation · Monascus purpureus · Pigment Monascus azaphilone · Triton X-100

Hyung-Jin Kwon $(\bowtie)$

E-mail:hjink@mju.ac.kr

${ }^{1}$ Department of Biological Sciences and Bioinformatics, Myongji University, Yongin-si, Gyunggi-do 17058, Republic of Korea

${ }^{2}$ Department of Oriental Medicine Resources and Institute for Traditional Korean Medicine Industry, Mokpo National University, Muan-gun, Jeollanam-do 58554, Republic of Korea

This is an Open Access article distributed under the terms of the Creative Commons Attribution Non-Commercial License (http://creativecommons. org/licenses/by-nc/3.0/) which permits unrestricted non-commercial use, distribution, and reproduction in any medium, provided the original work is properly cited.

\section{Introduction}

Monascus species, including M. anka, M. pilosus, M. purpureus and M. ruber, are used for food fermentation in eastern Asia [1]. The health benefits of Monascus are generally attributed to a polyketide metabolite, monacolin $\mathrm{K}$ (lovastatin), and it is often used for the long-term management hypercholesterolemia [2]. Monascus is also renowned for its high productivity of the pigment azaphilone (Az), a food colorant $[3,4]$. Azs comprise a class of fungal polyketides possessing an oxygenated pyranoquinone moiety [5]. The major members of Monascus Az (MAz) are ankaflavin (1), monascin (2), rubropunctatin (3), monascorubrin (4), rubropunctamine (5), and monascorubramine (6) (Fig. 1).

MAz components are generally classified by their colors: yellow, orange and red. The absorption peaks of the yellow ( 1 and 2), orange (3 and 4), and red (5 and 6) pigments are 390, 470, and $\sim 520 \mathrm{~nm}$, respectively, and the $\mathrm{pH}$ dependency of their visible light absorption pattern has been documented [3,6]. MAz red pigments also have an absorption maximum at $\sim 410 \mathrm{~nm}$. Structurally, $\mathbf{1}$ and $\mathbf{2}$ are in a reduced status by two hydride equivalents compared to $\mathbf{3}$ and $\mathbf{4}$, respectively (Fig. 1), which is the structural basis of their color difference. Biosynthetically, $\mathrm{MAz}$ is produced through a non-reducing polyketide pathway with the addition of fatty acyl moieties such as hexanoyl and octanoyl groups that are characteristic of $\mathbf{1 ; 3} ; \mathbf{5}$ and $\mathbf{2 ; 4} \mathbf{4} \mathbf{6}$, respectively $[7,8]$. Primary amine derivatives nonenzymatically convert $\mathbf{3}$ and $\mathbf{4}$ into their cognate versions of $\mathbf{5}$ and $\mathbf{6}$, respectively $[5,7]$. Therefore, finding diverse amino acid derivatives of $\mathbf{5}$ and 6 in Monascus extracts is not surprising [9]; Monascus is often called red yeast due to its characteristic red hue in rice fermentation products. The name azaphilone is derived from this azaphilic character, the tendency to aminate; while $\mathbf{1}$ and $\mathbf{2}$ are actually not azaphilic at all, they are routinely included in the $\mathrm{Az}$ group. The health benefits of MAz have been documented previously $[3,4]$, and reports of the disease alleviating activities of $\mathbf{1}$ and $\mathbf{2}$ have accumulated [10,11]. For orange MAz 3, anti-cancer potential and antioxidative effects have been reported [12-14]. 
The MAz biosynthetic gene cluster harbors three oxidoreductase genes $[7,15]$. The respective roles of these oxidoreductase genes have been elucidated within the scope of MAz biosynthesis [1618]. Two oxidoreductase $\mathrm{MppE}$ and $\mathrm{MppG}$ mediate the final divergent steps in MAz biosynthesis: $\mathrm{MppE}$ and $\mathrm{MppG}$ yield yellow and orange MAzs, respectively (Fig. 1). A hypothetical intermediate is hypothesized to be reduced by $\mathrm{MppE}$ to yield yellow congeners ( $\mathbf{1}$ and $\mathbf{2}$ ), while the MppG oxidase generates orange congeners (3 and $\mathbf{4})$.

Extractive fermentation (EF) is a general term for any fermentation with simultaneous product extraction. An evident advantage of EF is that it minimizes product inhibition inside cells. EF of a Monascus anka strain with the nonionic detergent Triton X-100 (TX100) was found to promote the export of MAzs, shifting the total MAz composition to a dominant yellow hue [1921]. TX100 was selected with its highest efficacy among various nonaqueous solvents tested [19]. TX100 was proposed to form micelles with MAzs, allowing these hydrophobic compounds to be soluble in aqueous media, thus promoting their export. This mechanism appears as a mere equilibrium shift. Later, TX100 was proposed to penetrate inside cells to extract MAzs stored in vacuoles [22]; however, how much this mode of action contributes to the export of MAzs is questionable as the micelle-forming effect is undoubtedly a major contributor to export.

The yellow MAz 1 and $\mathbf{2}$ congeners were initially suggested to selectively accumulated during TX100 EF by interpreting ultraviolet (UV)-visible absorption spectra and thin layer chromatography analyses [21]. However, these chemical analyses are incapable of revealing the identities of the yellow pigments under consideration. The supplementation of TX100 was recently reported to strongly induce novel yellow pigments from Monascus anka GIM 3.592, as determined by liquid chromatography (LC)-mass spectrometry (MS) analysis [23]. These yellow compounds have a UV-visible absorption peak at $430 \mathrm{~nm}$, but their structures are yet unknown. The deduced molecular weights of these yellow substances were much higher than those of MAz 1 to $\mathbf{6}$, which hampered predicting their structural relationship to these MAzs. No matter the origin of these yellow substances, the EF of Monascus evidently affects the MAz production profile by exporting them. Additional studies with Monascus anka provided some biological aspects of TX100 EF $[24,25]$, but no solid theory exists regarding how EF affects the MAz composition of yellow (1 and $\mathbf{2}$ ) and orange (3 and $\mathbf{4}$ ) components; the composition of the reduced and oxidized $\mathrm{MAz}$ congeners. Addressing this issue is the main purpose of this study.

Other than application of nonaqueous solvent, various EF methods are known and inclusion of macroporous absorbent resin is one of them. For non-polar metabolites, the polyaromatic absorbent resin Diaion HP-20 (HP20) is effective in promoting product secretion [26,27]. In this report, we demonstrated that supplementation with HP20 or TX100 was capable of promoting the production of orange MAz 3 and 4, (the oxidized MAz congeners), while suppressing the yield of yellow MAz $\mathbf{1}$ and $\mathbf{2}$, in a submerged culture of Monascus purpureus KACC (Korean Agricultural Culture Collection) 42430. It seems likely that a high export tendency of orange MAz over yellow MAz promotes the production of $\mathbf{3}$ and $\mathbf{4}$ in EF.

\section{Materials and Methods}

\section{Strains, culture conditions, and extraction methods}

Monascus purpureus KACC 42430 (DSM 1379) was used in this study. M. purpureus and its derivatives were cultured on potato dextrose agar (PDA) at $30{ }^{\circ} \mathrm{C}$ for 7 days. Agar blocks retrieved from the PDA culture were used to inoculate potato dextrose broth (PDB; Gellix ${ }^{\mathrm{TM}}$, Ventech Bio, Seoul, Republic of Korea). The liquid submerged cultures, prepared in $50 \mathrm{~mL}$ culture volume in a $250 \mathrm{~mL}$ baffled flask, were maintained in rotary shaker at $28{ }^{\circ} \mathrm{C}$ for 7 days. Two different agitation speeds were used in this study; 100 and $250 \mathrm{rpm}$. Ten milliliter of Diaion HP-20 resin (HP20; Samyang Co., Seoul, Korea) or $2.5 \mathrm{~mL}$ of Triton X-100 (TX100; Amresco, Solon, OH, USA) was added to the PDB media at the initiation of the culture for EF.

From the PDB culture, mycelia were separated from the supernatant by passing through a filter paper under reduced pressure. HP20 that was collected together with the mycelia during filtering was separated from mycelia by passing through a disposable net strainer (approximately $1 \mathrm{~mm}$ pore). By repeatedly applying water flow with a wash bottle, we made a majority of HP20 pass through the net strainer. We assumed that no less than 95\% HP20 was recovered this way. The mycelia pellets and HP20 were then extracted with methanol of the culture volume. Diethyl ether was the extraction choice for the supernatant from the TX100 culture [28]. The supernatant from the HP20 culture displayed no color at all and thus not processed in this experiment. The extract was evaporated under a reduced pressure and the residue was dissolved in methanol for chemical analyses.

\section{Chemical analysis methods}

UV-visible absorption spectra were collected with a Cary50 spectrophotometer (Varian, Palo Alto, CA, USA). The absorption spectra were collected with proper dilutions in methanol, and the values were converted to the corresponding values in the original culture volume. In measuring of UV-visible absorption, the culture supernatant was directly applied without extraction while the methanol extract was used for the cell. Orbitrap high-resolution LC (HPLC)-MS analysis was performed with Dionex UltiMate 3000 UHPLC (Thermo Scientific, Sunnyvale, CA, USA) combined with LTQ XL Orbitrap mass spectrometer (Thermo Scientific, Bremen, Germany) on a reverse-phase Kinetex HPLC column $(100 \mathrm{~mm} \times 2.1 \mathrm{~mm}$, particle size of $1.7 \mu \mathrm{m}$, pore size of $10 \mathrm{~nm}$; Phenomenex, Torrance, CA, USA), which was maintained at $40{ }^{\circ} \mathrm{C}$. The flow rate was maintained at $0.2 \mathrm{~mL} / \mathrm{min}$. Gradient elution was performed with the mobile phase composed of $0.1 \%$ 

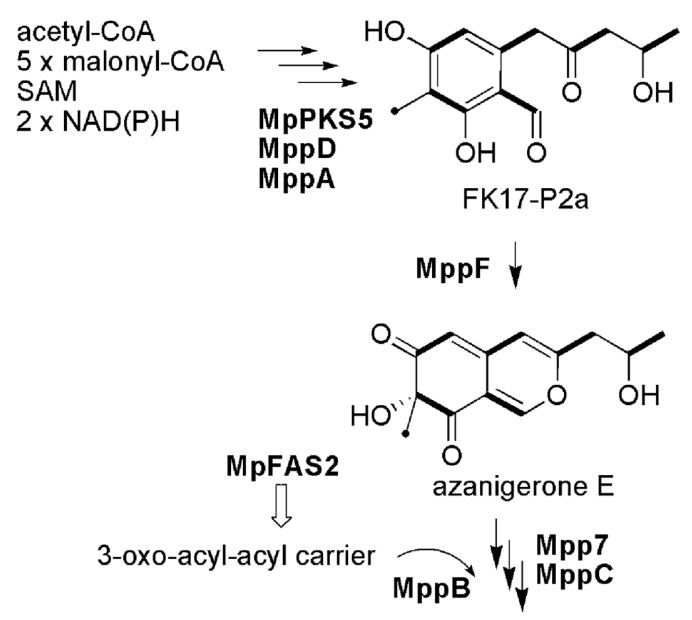

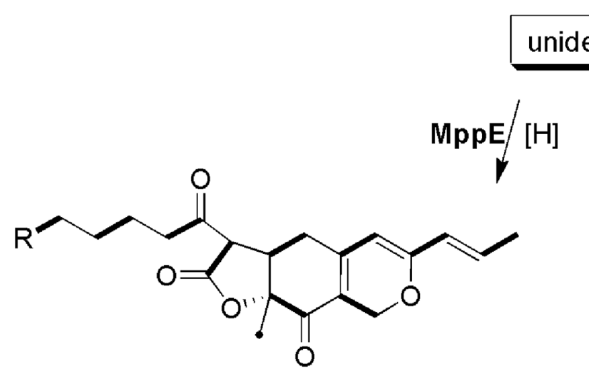

$1\left(\mathrm{R}=-\mathrm{CH}_{3}\right) ; 2\left(\mathrm{R}=-\left(\mathrm{CH}_{2}\right)_{2} \mathrm{CH}_{3}\right)$
[O] MppG<smiles>[R]CCCCC(=O)C1=C2C=C3C=C(C=CC)OC=C3C(=O)[C@@]2(C)OC1=O</smiles>

$3\left(\mathrm{R}=-\mathrm{CH}_{3}\right) ; 4\left(\mathrm{R}=-\left(\mathrm{CH}_{2}\right)_{2} \mathrm{CH}_{3}\right)$

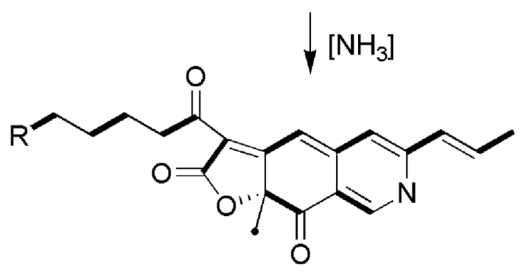

$5\left(\mathrm{R}=-\mathrm{CH}_{3}\right) ; 6\left(\mathrm{R}=-\left(\mathrm{CH}_{2}\right)_{2} \mathrm{CH}_{3}\right)$

Fig. 1 Structures of MAzs (1-6) with their proposed biosynthetic pathway. To emphasize their biosynthetic origins, the acetate units and $S$-adenosyl-Lmethionine-derived carbons are denoted with bars and black dots, respectively

formic acid in water (A) and $0.1 \%$ formic acid in acetonitrile (B). The HPLC gradient elution was applied with the following program: from $90 \%$ A to $50 \%$ A over $20 \mathrm{~min}$, from $50 \%$ A to $100 \% \mathrm{~B}$ over $10 \mathrm{~min}$, then maintained at $100 \% \mathrm{~B}$ for $10 \mathrm{~min}$. Electrospray ionization was performed in positive-ion mode and mass spectra were obtained in Fourier-transform mode with resolution of $\mathrm{R}=30,000$ at $\mathrm{m} / \mathrm{z} 400$. Metabolites were detected by full-scan mass analysis from $\mathrm{m} / \mathrm{z} 200$ to 500 , with data-dependent MS/MS analysis triggered by the most abundant ions from the precursor list. The collision energy was set to $35 \%$.

\section{Results and Discussion}

We investigated the effects of HP20 and TX10 on MAz production in $M$. purpureus in PDB submerged cultures. We aimed to evaluate the effects of these EF methods on the profile of major MAz components $\mathbf{1}$ to $\mathbf{4}$ in wild-type M. purpureus (WT) as well as two recombinant derivative strains, $\triangle m p p G$ and $\triangle m p p E$. The $\triangle m p p G$ and $\triangle m p p E$ strains selectively accumulate yellow (1 and 2) and orange (3 and 4) MAzs, respectively [17,18].

\section{EF resulted in a reduction of yellow MAzs 1 and 2}

Consistent with previous reports [21,23], TX100 promoted the export of MAzs into the culture supernatant and so did HP20. When HP20 was added, no pigment was found in the supernatant itself (data not shown); thus, the methanol eluate of the recovered HP20 represented all of the MAzs that were exported. LC-MS analysis was performed only with the extract that harbored the majority of MAzs in each experiment: the methanolic cell extract, the methanol eluate of the resin and the diethyl ether extract of the supernatant for the control, HP20 and TX100 cultures, respectively. 
(A)

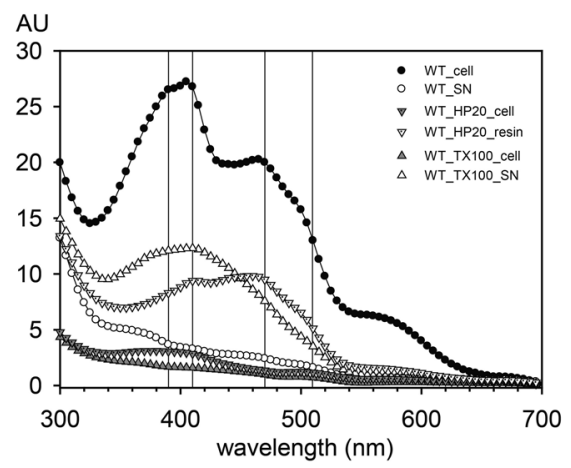

(B)
(C)

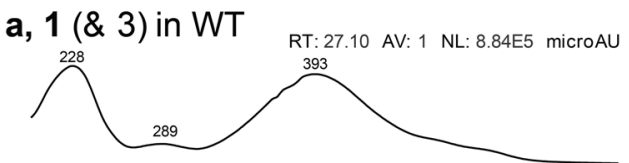

b, 1 \& 3 in WT_HP2O

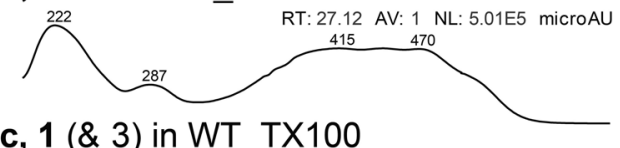

c, 1 (\& 3) in WT_TX100

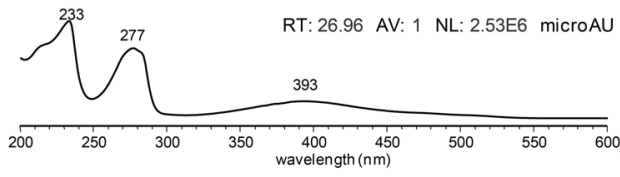

$1(\& 3)$

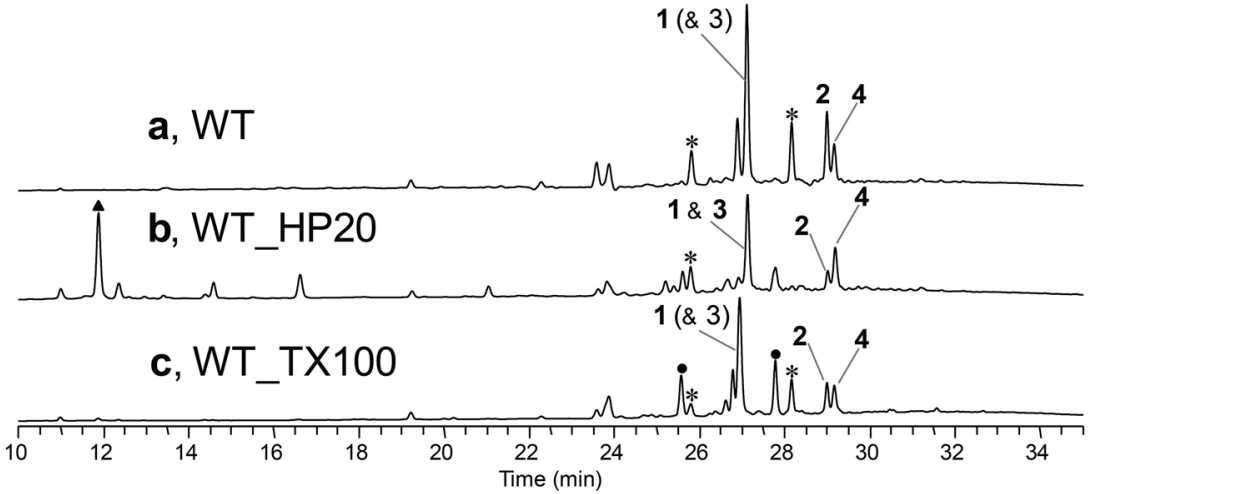

Fig. 2 Extractive fermentation suppressed the production of yellow MAzs in M. purpureus. (A) Absorption spectra of the control, HP20 and TX100 cultures are represented with circles, dotted down-pointing triangles and up-pointing triangles, respectively. The filled and unfilled symbols represent the cellular and supernatant extracts, respectively. Here, we regard the HP20 resin eluate as the supernatant extract. The wavelengths characteristic of the MAz components are indicated. (B) LC traces of the LC-MS data, monitored at $330 \mathrm{~nm}$ and drawn to the same Y-scale, are shown for the control/ cell extract (a, WT), the HP20/resin methanol eluate (b, WT_HP20) and the TX100/supernatant diethyl ether extract (c, WT_TX100). MAzs are indicated with their number annotations, while Y-1/Y-2 (the undefined yellow substances found in the TX100 culture), MC-1/MC-3 (the MAz derivatives from the $\triangle m p p C$ mutant) and FK17-P2a (the early intermediate of MAz) are indicated with circles, asterisks circles and triangles, respectively. (C) UV-visible absorption spectra of the peaks for $\mathbf{1}$ and $\mathbf{3}$ in the LC-MS analysis, of the control/cell extract (a, WT), the HP20/resin methanol eluate (b, WT_HP20) and the TX100/supernatant diethyl ether extract (c, WT_TX100) are shown to represent the relative abundances of 1 and $\mathbf{3}$

In WT, the overall absorbances of the TX100 and HP20 samples were lower than that of the control (Fig. 2A). The control sample displayed a broad absorption peak at a wavelength higher than $510 \mathrm{~nm}$, indicative of red components. Compared with the TX100 sample, which had high absorption at approximately 400 $\mathrm{nm}$, a broad absorption peak at $470 \mathrm{~nm}$ was evident in the HP20 sample. Compared to the control, a lower content of yellow compounds could be predicted for the HP20 sample.

LC-MS analysis was employed to support the presence of $\mathbf{1}$ to 4; chromatograms monitored at selected wavelengths (390, 470 and $520 \mathrm{~nm}$ for yellow, orange and red MAzs, respectively), with relevant mass spectra, are shown in Fig. S1. MC-1 and MC-3 are the MAzs isolated from the $\triangle m p p C$ mutant [16], and they are marked with asterisks in the LC chromatograms (Fig. 2B). Among the four MAzs of the $\triangle m p p C$ mutant, both MC-1 and MC-3 belong to the reduced class (Fig. 1). The presence of red MAzs could be found only in the control, as seen in monitoring at $520 \mathrm{~nm}$ (Fig. S1). This finding could be due to the immediate export of $\mathbf{3}$ and $\mathbf{4}$, which minimizes the formation of red MAzs. The red MAzs were previously suggested to form that red MAz is formed inside the cell through the reaction of intracellular amine derivatives [29]. Under these LC conditions, the peaks for $\mathbf{1}$ and $\mathbf{3}$ overlapped; thus, UV-visible absorption and mass spectra were used to deduce the relative compositions of $\mathbf{1}$ and $\mathbf{3}$. When compared to the control, the HP20 sample displayed a reduction in the yellow MAzs 1 and 2 (Fig. 2B). A relative enrichment of 3 (versus 1) in the HP sample was evident in the UV-visible absorption spectrum (Fig. 2C) as well as the mass spectrum of the cognate elution peak (Fig. S1B). In terms of a reduction of yellow MAzs, These LC-MS data are consistent with the UV-visible spectra data in Fig. 2A. A peak unique to the HP20 sample was that for FK17-P2a (marked as triangles), which was previously established as the early intermediate in MAz biosynthesis (Fig. 1) [16]. This finding suggests that the capacity of HP20 to trap 
(A)

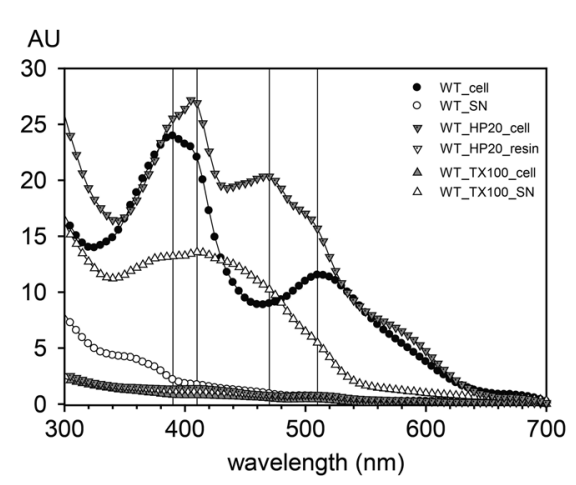

(C)

\section{a, 1 in WT}

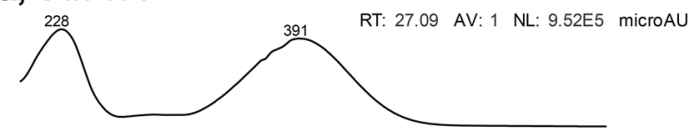

b, 1 \& 3 in WT_HP20

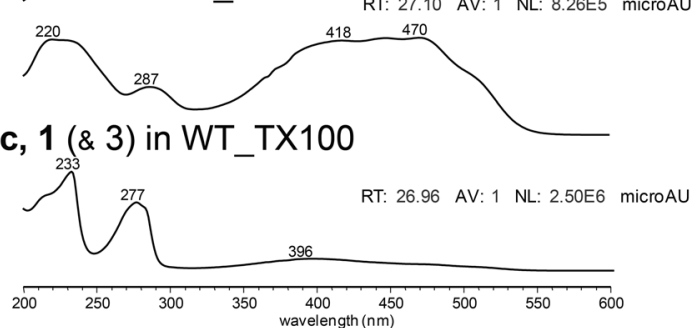

(B)

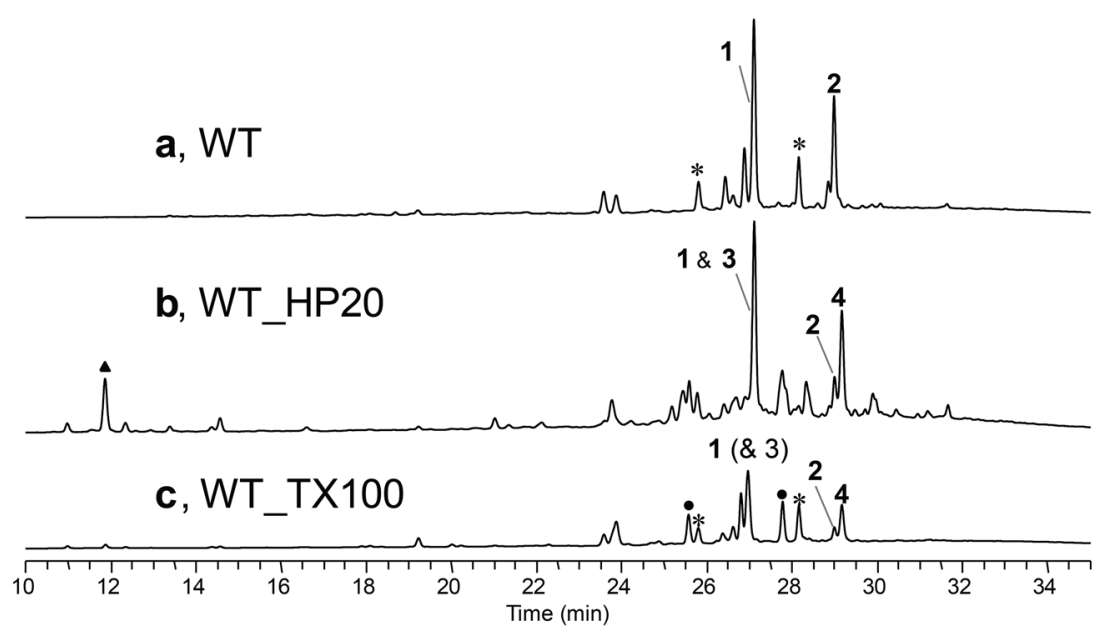

Fig. 3 Extractive fermentation promoted the production of orange MAzs, while inhibiting that of yellow MAzs, in M. purpureus when cultured at 100rpm agitation. (A) Absorption spectra are shown with the symbol choice used in Fig. 2. (B) LC traces of the LC-MS data, monitored at $330 \mathrm{~nm}$ and drawn to the same Y-scale, are shown for the control/cell extract (a, WT), the HP20/resin methanol eluate (b, WT_HP20) and the TX100/supernatant diethyl ether extract (c, WT_TX100). Compound marking is the same as Fig. 2. (C) UV-visible absorption spectra of the peaks for 1 and 3 in the LCMS analysis, of the control/cell extract (a, WT), the HP20/resin methanol eluate (b, WT_HP20) and the TX100/supernatant diethyl ether extract (c, WT_TX100) are shown to represent the relative abundances of $\mathbf{1}$ and $\mathbf{3}$

hydrophobic compounds is capable of driving the export of this biosynthetic intermediate, which does not accumulate during cellular biosynthesis. This type of export mechanism appeared to enhance the production of FK17-P2a. This phenomenon did not occur significantly when TX100 was used under these conditions. We also found a reduction in yellow MAz 2 in the TX100 sample (Fig. 2B). The TX100 sample contained two yellow peaks (marked as circles) other than $\mathbf{1}$ and $\mathbf{2}$ (Fig. 2B). Their absorption maxima are at $430 \mathrm{~nm}$ (data not shown), implying that they are the yellow pigments reported from the TX100 EF of Monascus anka GIM 3.592 [23]. For convenience, these two compounds will be referred Y-1 and Y-2. Oddly, multiple ion peaks were found for the Y-1 and Y-2 peaks, hampering the identification of relevant molecular ion peaks in this experiment (Fig. S1C). An obstacle to analyzing the TX100 samples was that residual TX100 eluted with $\mathbf{1}$ and $\mathbf{3}$, causing a shift in their retention times (Fig. 2B and C). Furthermore, the molecular ion peaks of $\mathbf{1}$ and $\mathbf{3}$ could not be found in the LC-MS traces of the TX100 sample; thus, TX100 was assumed to suppress the ion peaks for $\mathbf{1}$ and $\mathbf{3}$. In fact, all the mass spectra from the TX100 sample contained several unknown peaks, and we could not determine the origin of these peaks (Fig. S1C). LC traces suggested the presence of Y-1 and Y2 in the HP20 sample (Fig. 2B), but the chemical information, such as UV-visible absorption patterns and characteristics of the MS data, was not convincing of their presence. This LC-MS pattern was also observed in the HP20 samples of other culture experiments, as shown below. Neither HP20 nor TX100 appeared capable of promoting overall MAz productivity during the PDB culture of $M$. purpureus, whereas reductions in $\mathbf{1}$ and $\mathbf{2}$ were evident with either HP20 or TX100 (Fig. 2).

For the $\triangle m p p G$ mutant, a reduction in UV-visible absorption of the HP20 sample was more pronounced than it was for the WT (Fig. S2). Otherwise, the outcomes of EF were comparable between the WT and $\Delta m p p G$ strains. As seen in the WT strain, the 
(A)

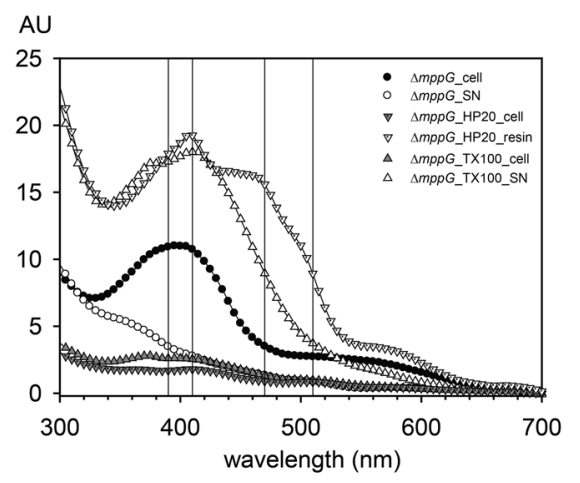

(B)
(C)

\section{a, 1 in $\triangle m p p G$}

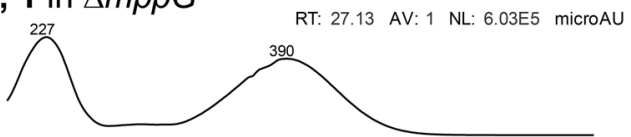

b, 1 \& 3 in $\triangle m p p G \_H P 20$

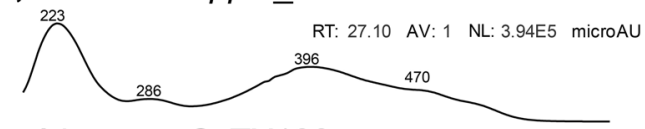

c, 1 in $\triangle m p p G \_T X 100$

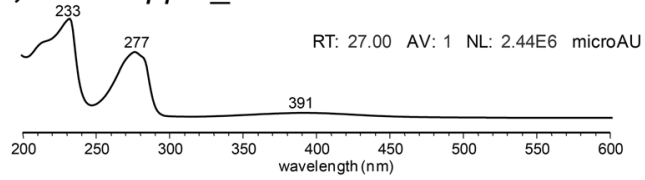

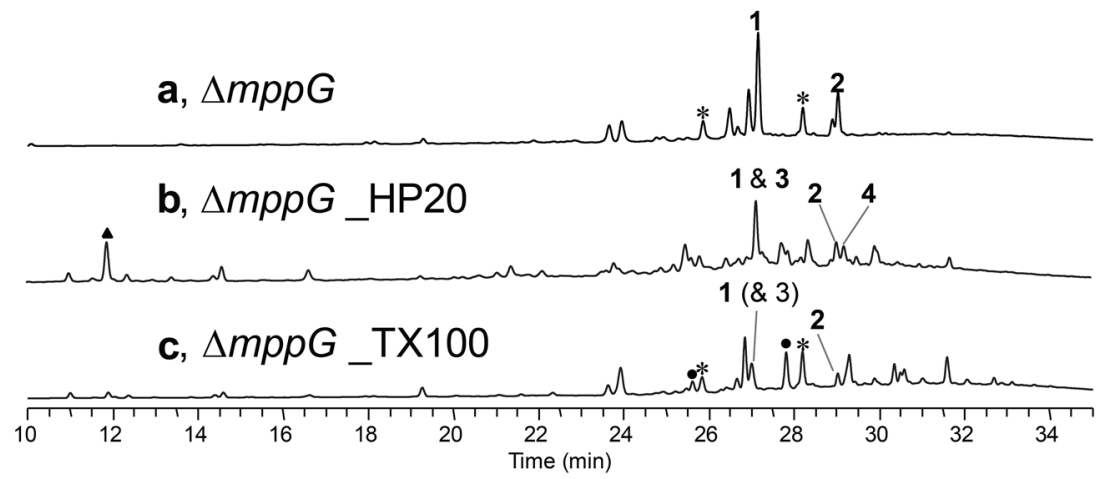

Fig. 4 Extractive fermentation promoted the production of orange MAzs, while inhibiting that of yellow MAzs, in $M$. purpureus $\Delta m p p G$ mutant when cultured at 100-rpm agitation. (A) Absorption spectra are shown with the symbol choice used in Fig. 2. (B) LC traces of the LC-MS data, monitored at $330 \mathrm{~nm}$ and drawn to the same Y-scale, are shown for the control/cell extract (a, $\Delta \boldsymbol{m p p} \boldsymbol{G})$, the HP20/resin methanol eluate (b, $\left.\Delta \boldsymbol{m p p} \boldsymbol{G} \_\mathbf{H P 2 0}\right)$ and the

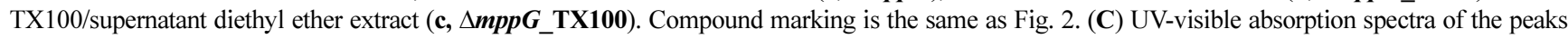
for $\mathbf{1}$ and $\mathbf{3}$ in the LC-MS analysis, of the control/cell extract (a, $\Delta \boldsymbol{m p p} \boldsymbol{G})$, the HP20/the resin methanol eluate (b, $\Delta \boldsymbol{m p p} \boldsymbol{G}$ HP20) and TX100/the supernatant diethyl ether extract (c, $\Delta m p p G_{-}$TX100) are shown to represent the relative abundances of 1 and 3

red component content decreased in both of the HP20 and TX100 samples, while the absorption near $400 \mathrm{~nm}$ decreased in the HP20 sample (Fig. S2A). LC-MS analysis found 1, 2, MC-1 and MC3 in the control for $\triangle m p p G$, and $\mathbf{1}$ and $\mathbf{2}$ were found to decrease in both EF samples (Fig. S2B and C). Y-1 and Y-2 (circles) were also found in the TX100 sample (Fig. S2B). These chromatograms, monitored at selected wavelengths with relevant mass spectra, are shown in Fig. S3.

For the $\triangle m p p E$ mutant, all the samples displayed a dominance of orange MAz 3 and 4 (Fig. S4), and severe growth retardation was observed with HP20; approximately $2 \mathrm{mg} / \mathrm{mL}$ wet cell weight was obtained when HP20 was added, while the control yielded 25 $\mathrm{mg} / \mathrm{mL}$. With this low growth, the MAz yield of the HP20 sample was significantly lower than that of the control (Fig. S4A). The TX100 sample afforded MAzs at a level comparable to that of the control and displayed an absorption peak at approximately 430 $\mathrm{nm}$. LC-MS analysis demonstrated that the levels of $\mathbf{3}$ and $\mathbf{4}$ were comparable between the control and the TX100 samples. A high level of Y-1 and Y-2 (circles) were found in the TX100 samples of the $\triangle m p p E$ strain (Fig. S4B). These chromatograms, monitored at selected wavelengths with relevant mass spectra, are shown in Fig. S5. The HP20 sample contained R-1 and R-2, the ethanolamine derivatives of red MAz 5 and $\mathbf{6}$, respectively. These compounds were previously identified in the $M$. purpureus $\triangle m p p E$ and $\triangle m p p D E G$ mutants [29]. Low yields of $\mathbf{3}$ and $\mathbf{4}$ might make $\mathbf{R}$ $\mathbf{1}$ and R-2 noticeable in the chromatograms.

In summary, EF with either HP20 or TX100 resulted in a reduction of $\mathbf{1}$ and $\mathbf{2}$ during $\mathrm{PDB}$ culture with the M. purpureus strains used in this study (Fig. 2 and S2). As reported in a M. anka strain [23], unknown yellow compounds Y-1 and Y-2 were found when TX100 was added, but these compounds were not observed when HP20 was used (Fig. 2B, S2B and S4B), implying that the formation of Y-1 and Y-2 is related to TX100 itself and not to the MAz export. The shift to a yellow hue that was observed in the TX100 culture of a $M$. anka strain [21] could result from the production of yellow compounds related to Y-1 and Y-2. Considering that these unknown yellow substances are structurally undefined, the practical significance of Monascus EF with TX100 seems doubtable. Our attention was drawn to the production of FK17P2a in the HP20 samples (Fig. 2B, S2B and S4B). This compound 
(A)

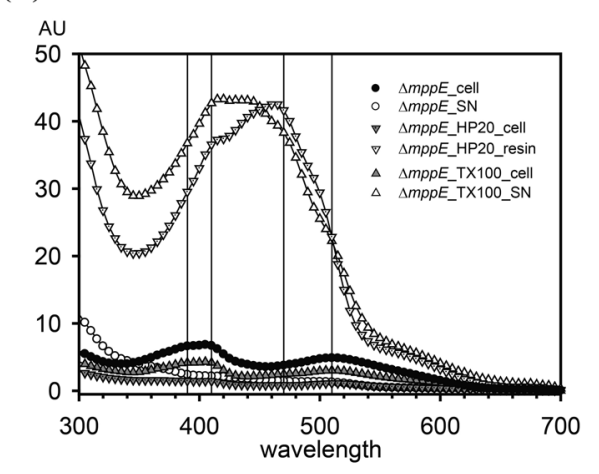

(B)

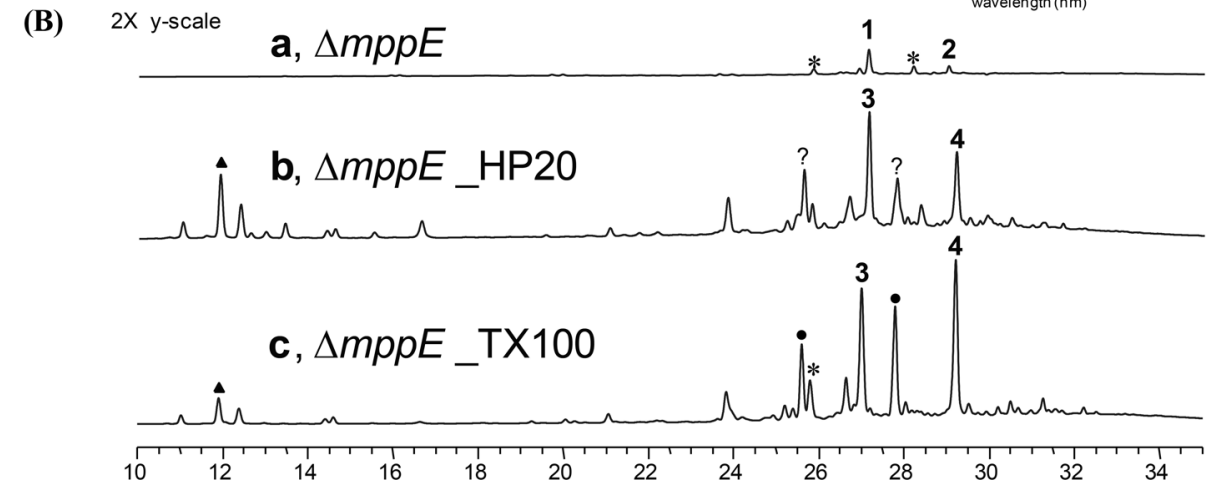

(C)

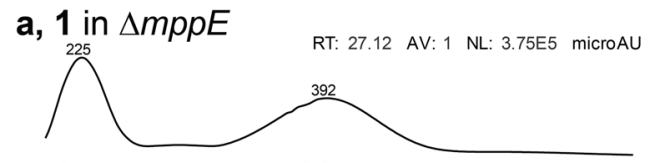

b, 3 in $\triangle m p p E \_H P 20$ RT: 27.12 AV: 1 NL: 1.14E6 microAU

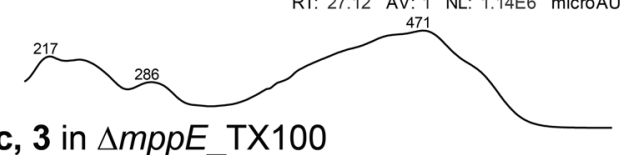

c, 3 in $\triangle m p p E \_T X 100$

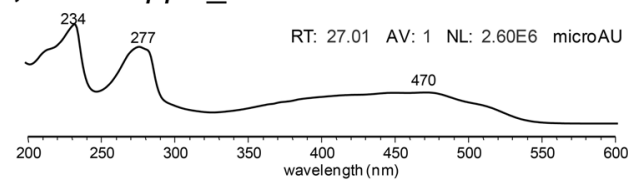

Fig. 5 Extractive fermentation promoted the production of orange MAzs in M. purpureus $\Delta m p p E$ mutant when cultured at 100-rpm agitation. (A) Absorption spectra are shown with the symbol choice used in Fig. 2. (B) LC traces of the LC-MS data, monitored at 330 nm and drawn to the same Yscale, are shown for the control/cell extract (a, $\Delta \boldsymbol{m p p E}$ ), the HP20/resin methanol eluate (b, $\Delta \boldsymbol{m p p E}$ HP20) and the TX100/the supernatant diethyl ether extract (c, $\Delta \boldsymbol{m p p E} \boldsymbol{E}_{-} \mathbf{T X 1 0 0}$ ). The absolute Y-scale is as two times as those of Fig. 3 and 4. Compound marking is the same as Fig. 2. (C) UVvisible absorption spectra of the peaks for $\mathbf{1}$ and $\mathbf{3}$ in LC-MS analysis, of the control/cell extract (a, $\Delta \boldsymbol{m p p E}$ ), the HP20/resin methanol eluate (b, $\left.\Delta \boldsymbol{m p p E \_ H P 2 0}\right)$ and the TX100/supernatant diethyl ether extract (c, $\left.\Delta \boldsymbol{m p p} \boldsymbol{E}_{-} \mathbf{T X 1 0 0}\right)$ are shown to represent the relative abundances of $\mathbf{1}$ and $\mathbf{3}$

is an early intermediate released from the MAz polyketide synthase MpPKS5, and it specifically accumulates in the $\triangle m p p F$ mutant (Fig. 1), which lacks the gene encoding the hydroxylase that converts FK17-P2a into azanigerone E [16]. The hydroxylated derivative of FK17-P2a is nonenzymatically cyclized to give azanigerone E [30]. This finding implies that the HP20 is capable of inducing or enhancing the production of intermediate(s) by promoting its export, at least for Az biosynthesis.

EF promotes orange MAz production at a low agitation speed In the following evaluation of EF, we employed low-speed agitation during culturing, which afforded MAz profiles different from those of cultures agitated at $250 \mathrm{rpm}$. Notably, the supplementation of HP20 exerted no inhibitory effects on the growth of the $\triangle m p p E$ mutant when cultured at 100 -rpm agitation, unlike the high-agitation culture. In a 100-rpm culture, WT mainly produced yellow MAz 1 and $\mathbf{2}$ and the addition of HP20 not only reduced the levels of $\mathbf{1}$ and $\mathbf{2}$ but also enhanced those of $\mathbf{3}$ and $\mathbf{4}$ (Fig. 3), interestingly switching the MAz profile from a dominance of yellow MAzs to orange ones. For the TX100 samples, the MAz profiles appeared comparable to those obtained in the $250-\mathrm{rpm}$ experiment (Fig. 2 and 3). These chromatograms, monitored at selected wavelengths with relevant mass spectra, are shown in Fig. S6. For the $\triangle m p p G$ mutant, the overall pigment content was significantly enhanced via both EF methods, with evident enhancement in absorbance at $470 \mathrm{~nm}$ in the HP20 sample (Fig. 4A). LC-MS analysis also supported a reduction in yellow MAzs with a concomitant enhancement in orange MAzs 3 and 4 (Fig. 4B and $\mathrm{C}$ ). These chromatograms, monitored at selected wavelengths, with relevant mass spectra, are shown in Fig. S7.

The $\triangle m p p E$ mutant, which produced mainly orange MAz with high agitation, displayed low MAz productivity in low-agitation culture with an undetectable level of orange MAzs (Fig. 5A and B). The $\triangle m p p E$ mutant was previously established to preferentially produce orange MAzs over yellow ones [17]. The present study demonstrated that low-agitation culturing conditions limited orange MAz production. These two factors might give rise to the dramatic reduction in MAz productivity observed for $\triangle m p p E$ in low-agitation culture. In these circumstances, a strong enrichment of $\mathbf{3}$ and $\mathbf{4}$ production was observed with both HP20 and TX100 (Fig. 5A). LC-MS analysis supported the selective production of 3 and 4, as well as FK17-P2a, under both EF conditions (Fig. 5B 
and C). These chromatograms, monitored at selected wavelengths with relevant mass spectra, are shown in Fig. S8.

These low-agitation culture experiments demonstrated that both HP20 and TX100 EF promote the production of orange MAzs 3 and 4 in $M$. purpureus (Figs. 3-5). It seems likely that the chemical properties of the oxidized MAzs $\mathbf{3}$ and $\mathbf{4}$ have a high tendency of passing through the cell barrier, over the reduced MAzs 1 and 2, and EF promotes the export by either solubilizing (with TX100) or sequestering (with HP20) the exported compounds. This action EF gives rise to an equilibrium shift in favor of the exported. We propose that the change of MAzs composition in EF originated from a difference of tendency passing the cell barrier, although it is yet to be defined whether this tendency is a mere reflection of the chemical nature or involves any cellular transport system. This observation highlights the possibility that EF modulates the MAz profile in favor of oxidized MAz congeners, contrasting with previous theories that the TX100 EF of Monascus promotes yellow MAzs [21] and revealing the significance of the present study.

Acknowledgment This research was supported by Basic Science Research Program through the National Research Foundation of Korea (NRF) funded by the Ministry of Education (NRF-2016R1D1A1B02009237).

\section{References}

1. Wang TH, Lin TF (2007) Monascus rice products. Adv Food Nutr Res 53: $123-159$

2. Manzoni M, Rollini M (2002) Biosynthesis and biotechnological production of statins by filamentous fungi and application of these cholesterol-lowering drugs. Appl Microbiol Biotechnol 58: 555-564

3. Feng Y, Shao Y, Chen F (2012) Monascus pigments. Appl Microbiol Biotechnol 96: 1421-1440

4. Patakova P (2013) Monascus secondary metabolites: production and biological activity. J Ind Microbiol Biotechnol 40: 169-181

5. Gao JM, Yang SX, Qin JC (2013) Azaphilones: chemistry and biology. Chem Rev 113: 4755-4811

6. Shi K, Chen G, Pistolozzi M, Xia F, Wu Z (2016) Improved analysis of Monascus pigments based on their $\mathrm{pH}$-sensitive UV-Vis absorption and reactivity properties. Food Addit Contam Part A Chem Anal Control Expo Risk Assess 33: 1396-1401

7. Balakrishnan B. Karki S, Chiu SH, Kim HJ, Suh JW, Nam B, Yoon YM, Chen CC, Kwon HJ (2013) Genetic localization and in vivo characterization of a Monascus azaphilone pigment biosynthetic gene cluster. Appl Microbiol Biotechnol 97: 6337-6345

8. Balakrishnan B, Kim HJ, Suh JW, Chen CC, Liu KH, Park SH, Kwon HJ (2014) Monascus azaphilone pigment biosynthesis employs a dedicated fatty acid synthase for short chain fatty acyl moieties. J Kor Soc Appl Biol Chem 57: 191-196

9. Jung H, Kim C, Kim K, Shin CS (2003) Color characteristics of Monascus pigments derived by fermentation with various amino acids. J Agric Food Chem 51: 1302-1306

10. Lin CH, Lin TH, Pan TM (2017) Alleviation of metabolic syndrome by monascin and ankaflavin: the perspective of Monascus functional foods. Food Funct 8: 2102-2109

11. Liu SF, Wang YR, Shen YC, Chen CL, Huang CN, Pan TM, Wang CK
(2018) A randomized, double-blind clinical study of the effects of Ankascin 568 plus on blood lipid regulation. J Food Drug Anal 26: 393400

12. Zheng Y, Xin Y, Shi X, Guo Y (2010) Anti-cancer effect of rubropunctatin against human gastric carcinoma cells BGC-823. Appl Microbiol Biotechnol 88: 1169-1177

13. Zheng Y, Zhang Y, Chen D, Chen H, Lin L, Zheng C, Guo Y (2016) Monascus pigment rubropunctatin: a potential dual agent for cancer chemotherapy and phototherapy J Agric Food Chem 64: 2541-2548

14. Dhale MA, Javagal M, Puttananjaiah MH (2018) Protective and antioxidative effect of rubropunctatin against oxidative protein damage induced by metal catalyzed reaction. Int J Biol Macromol 116: 409-416

15. Balakrishnan B, Chen CC, Pan TM, Kwon HJ (2014) Mpp7 controls regioselective Knoevenagel condensation during the biosynthesis of Monascus azaphilone pigments. Tetrahedron Lett 55: 1640-1643

16. Bijinu B, Suh JW, Park SH, Kwon HJ (2014) Delineating Monascus azaphilone pigment biosynthesis: oxidoreductive modifications determine the ring cyclization pattern in azaphilone biosynthesis. RSC Adv 4: 59405-59408

17. Balakrishnan B, Park SH, Kwon HJ (2017) A reductase gene $m p p E$ controls yellow component production in azaphilone polyketide pathway of Monascus. Biotechnol lett 39: 163-169

18. Balakrishnan B, Park SH, Kwon HJ (2017) Inactivation of the oxidase gene $m p p G$ results in the selective loss of orange azaphilone pigments in Monascus purpureus. Appl Biol Chem 60: 437-446

19. Hu Z, Zhang X, Wu Z, Qi H, Wang Z (2012) Perstraction of intracellular pigments by submerged cultivation of Monascus in nonionic surfactant micelle aqueous solution. Appl Microbiol Biotechnol 94: 81-89

20. Kang B, Zhang X, Wu Z, Qi H, Wang Z (2013) Effect of pH and nonionic surfactant on profile of intracellular and extracellular Monascus pigments. Process Biochem 48: 759-767

21. Xiong X, Zhang X, Wu Z, Wang Z (2015) Accumulation of yellow Monascus pigments by extractive fermentation in nonionic surfactant micelle aqueous solution. Appl Microbiol Biotechnol 99: 1173-1180

22. Chen G, Tang R, Tian X, Qin P, Wu Z (2017) Change of Monascus pigment metabolism and secretion in different extractive fermentation process. Bioprocess Biosyst Eng 40: 857-866

23. Shi K, Tang R, Huang T, Wang L, Wu Z (2017) Pigment fingerprint profile during extractive fermentation with Monascus anka GIM 3.592. BMC Biotechnol 17:46. doi: 10.1186/s12896-017-0366-1

24. Chen G, Bei Q, Shi K, Tian X, Wu Z (2017) Saturation effect and transmembrane conversion of Monascus pigment in nonionic surfactant aqueous solution. AMB Express 7:24. doi:10.1186/s13568-017-0327-z

25. Chen G, Bei Q, Huang T, Wu Z (2017) Tracking of pigment accumulation and secretion in extractive fermentation of Monascus anka GIM 3.592. Microb Cell Fact 16:172. doi:10.1186/s12934-017-0786-6

26. Hara M, Asano K, Kawamoto I, Takiguchi T, Katsumata S, Takahashi K, Nakano H (1989) Leinamycin, a new antitumor antibiotic from Streptomyces, producing organism, fermentation and isolation. J Antibiot 42: 1768-1773

27. Singh MP, Leighton MM, Barbieri LR, Roll DM, Urbance SE, Hoshan L, McDonald LA (2010) Fermentative production of self-toxic fungal secondary metabolites. J Ind Microbiol Biotechnol 37: 335-340

28. Shen L, Zhang X, Liu M, Wang Z (2014) Microemulsion extraction of Monascus pigments from nonionic surfactant using high polarity of diethyl ether as excess oil phase. Sep Sci Technol 49: 2346-2351

29. Balarishnan B, Lim YJ, Hwang SH, Lee DW, Park SH, Kwon HJ (2017) Selective production of red azaphilone pigments in a Monascus purpureus mppDEG deletion mutant. J Appl Biol Chem 60: 249-256

30. Zabala AO, Xu W, Chooi YH, Tang Y (2012) Characterization of a silent azaphilone gene cluster from Aspergillus niger ATCC 1015 reveals a hydroxylation-mediated pyran-ring formation. Chem Biol 19: 10491059 